Journal of

Dentistry and Oral Health

\title{
Evaluation of Patient Satisfaction with Dentures Retained By Ceramic Primary and Electroplated Secondary Conical Double Crowns upto 16 Years
}

\section{Silvia Brandt ${ }^{1, *}$, Jan Brandt ${ }^{1}$, Hans- Christoph Lauer ${ }^{1}$, Anna Winter ${ }^{2}$}

${ }^{1}$ Department of Prosthodontics, School of Dentistry, Goethe University, Frankfurt am Main, Germany; ZZMK Carolinum, Theodor-Stern-Kai 7, Haus 29, 60590 Frankfurt am Main

${ }^{2}$ Department of Prosthodontics, School of Dentistry, Julius Maximilians University, Würzburg, Germany; Zentrum für Zahn-, Mund- und Kiefergesundheit, Pleicherwall 2, 97070 Würzburg

${ }^{*}$ Corresponding author: Silvia Brandt, Department of Prosthodontics, School of Dentistry, Goethe University, Frankfurt am Main, Germany; ZZMK Carolinum, Theodor-Stern-Kai 7, Haus 29, 60590 Frankfurt am Main; Tel: 317-278-6210; Email: hajjaj@med.uni-frankfurt.de

Received Date: October 20, 2018; Accepted Date: November 07, 2018; Published Date: November 09, 2018

Citation: Silvia Brandt (2018)Evaluation of Patient Satisfaction with Dentures Retained By Ceramic Primary and Electroplated Secondary Conical Double Crowns upto 16 Years. J Dent Oral Health 5: 1-6.

\footnotetext{
Abstract

Purpose: The purpose of this article is to evaluate patient satisfaction with dentures retained by ceramic primary and electroplated secondary conical double crowns (CEP), thus extending the coverage of the current literature in this field.

Materialsand methods: A total of 74 patients who had been treated with CEPbetween 1998 and 2013 were surveyed.Parameters like overall satisfaction, handling, wearing comfort and functional aspects get evaluated. Accordingto the location of the dentures, the patients were divided into three patient groups.

Results: The statistical analysis showed no significant influence of the location of the prosthesis (maxilla/mandible) on the parameters examined. The patient evaluations showed a high level of patient satisfaction with their prostheses.

Conclusion: Based on the results obtained, CEP for oral rehabilitation can be recommended from the dentist's and the patient's point of view if a clinical indication exists.

Abbreviations: MX : maxilla; MD: mandible; MX+MD: maxilla and mandible; VAS: visual analogue scale; CEP: denture retained by ceramic primary and electroplated secondary conical double crowns according to the Weigl protocol (ceramic/ electroplated double-crown prosthesis); OHRQoL: oral health-related quality of life.
}

C2018 The Authors. Published by the JScholar under the terms of the Creative Commons Attribution License http://creativecommons.org/licenses/by/3.0/, which loize.marechal@umontreal.ca

maximilien.laviolette-brassard@umontreal.ca 


\section{Introduction}

Treatment protocols and manufacturing methods are a major focus in scientific research aimed at evaluating and continuously improving dental treatment methods. Often, however, the perspective studied is that of the dentist, while the effect of the treatment on the patient and the ensuing changes have rarely been examined.

More recently, however, interest in patients' overall satisfaction and quality of life following dental procedures has increased. In this context, Montero et al. reviewed the impact of dental treatment on patients' oral health-related quality of life (OHRQoL). They found that the more negative the pretreatment impairment had been felt, the more pronounced were the perceived improvements in OHRQoL after dental rehabilitation [15].

In addition, Tan et al. and Gerritsen et al. demonstrated that the number and location of lost teeth also influenced OHRQoL $[13,19]$. Thus, the loss of many or even all teeth may restrict chewing comfort, esthetics, and speech function [22]. Especially in edentulous patients, implants supporting a dental prosthesis can improve patient satisfaction, chewing comfort, and the associated quality of life $[2,4,6,20]$. Preciado et al. found that the extent to which patient satisfaction and OHRQoL are improved by implant-supported prostheses depended on the retention mechanism used [17]. Awad et al. and Sánchez-Siles et al. reported that prostheses with bar attachments significantly improved OHRQoL compared to conventional prostheses $[3,18]$. Ellis et al. and Ceruti et al. reported that a similar improvement can be achieved with ball retainers and with magnets $[8,11]$. With a reduced number of 2 to 6 abutments, prostheses retained by telescope crowns also significantly improved OHRQoL [14].

The studies referred to thus examined and described the correlations between patient satisfaction with different overdenture retention systems. No reports, however, have been published on patient satisfaction with prostheses retained by ceramic primary and electroplated secondary conical double crowns (CEP). Here, the manufacturing process allows a gap of $5 \mu \mathrm{m}$ between primary and secondary crown. In contact with saliva, the tribological effect facilitates the retention of these dentures.

The purpose of the present article has been to evaluate the effect of these CEP on patient satisfaction, thus extending the coverage of the current literature in this field.

\section{Materials and Methods}

To determine eligibility, 74 patients were interviewed who had received a total of 87 CEPs according to the Weigl protocol [23] at the Department of Prosthodontics, School of Dentistry, Goethe University Frankfurt am Main, between 1998 and 2013.
In contrast to a double crown reatined denture with a conical or telescopic crown design, these dentures do not exhibit friction between its primary and secondary crowns. The manufacturing process allows a gap of $5 \mu \mathrm{m}$ between the primary and secondary crowns that serves as a capillary gap. In contact with saliva, this gap facilitates cohesive forces and steady retention of the denture. Thus, the retention principle is tribological in nature, with surfaces interacting in relative motion. Thereby there's no need for friction adjustments or even a loss or change in retention is expected during the lifetime of the denture. Furthermore another advantage of the conical electroplated dentures is owed to the design and material selection of the primary crowns. Here, the use of zirconia for primary crown production reduces the demasking effect.

The evaluated dentures were tooth-, implant and tooth-implant- supported without any support of the mucosa. The overall number amounts 403abutments (145 teeth, 258 implants). The mean number shows 5,7 abutments in the upper jaw and 3,9 abutments in the lower jaw. Furthermore 48 (14 MX, $34 \mathrm{MD}$ ) prostheses were implant-supported, 22 (12 MX, $10 \mathrm{MD})$ tooth-supported and 17 (9 MX, $8 \mathrm{MD})$ combined tooth-and implant supported.

Exclusion criteria were non-consent, malignant disease, and severe mental illness. In addition, acute dental treatment needs also precluded participation in the study. Inclusion criteria for the study were patient consent, prior oral rehabilitation with a CEP according to the Weigl protocol during the above-mentioned period, and compliance with annual follow-up evaluations at the Department of Prosthodontics. As part of a prior regular follow-up examination in 2014, patients had been informed about the project orally and received a questionnaire after having agreed to participate in the study. Based on this questionnaire, various parameters were evaluated that had an influence on the OHRQoL and patients' satisfaction with their prostheses. As has been common practice in comparable studies, visual analog scales (VAS) were used for the evaluation $[1,9,12,16]$.

The evaluation comprised the parameters Overall satisfaction, Handling, Retention, Wearing comfort, Esthetics, Chewing function, Stability, Sense of security, and Speech function as related to the CEP studied. The VAS used were $100 \mathrm{~mm}$ in length, with values ranging from 0 to $100(1 \mathrm{~mm}=$ $1 \%)$, where 0 denotes the "lowest" or "worst" limit and 100 the "highest" or "best" limit for the respective parameter. In addition, categorical questions were asked that could be answered "no," "neither-nor," or "yes." These were used to elicit patient responses for the parameters General problems, Changes since delivery, and Movement during chewing and speaking.

Once the patients had completed the evaluation, the investigator analyzed the questionnaires. The quantitative (VAS) results were tested using the Shapiro-Wilk test in order to examine possible signficiant differences for normal distribution. These data were then subjected to an analysis of variance. Fisher's exact test was used to evaluate the categorical results. The level of significance was set at $\mathrm{p} \leq 0.05$. The cal- 
culations were made using the IBM SPSS Statistics 24 software package (SPSS, Chicago, IL, USA).

The study had previously been approved by the competent Ethics Commission.

\section{Results}

Of the 74 patients who had consented to participate in the study and received and completed the questionnaire, 22 patients wore a maxillary prosthesis, 39 wore a mandibular prosthesis, and 13 wore prostheses in both jaws, for a total of 87 prostheses evaluated. This resulted in three patient groups with prostheses in the maxilla and mandible $(\mathrm{MX}+\mathrm{MD})$ a prosthesis in the maxilla only (MX only), and a prosthesis in the mandible only (MD only).

There were 36 male and 38 female participants.

Table 1 presents an overview of the VAS scores. Figure 1 illustrates the results for the Overall satisfaction parameter.

For the parameters considered, analyses of variance yielded no significant differences between the three patient groups regarding the location of the prosthesis ( $\mathrm{MX}+\mathrm{MD}, \mathrm{MX}$ only, MD only).Categorical questions were asked for General problems, Changes since delivery, and Movement during chewing and speaking. The overall assessment of these parameters is based on the responses within the three patient groups (MX+MD, MX only, MD only) The answer "no" was chosen by 61 patients each for General problems and Changes since delivery and by 67 patients for Movement during chewing and speaking. The answer "neither-nor" was chosen by 12 patients for General problems, by 11 patients for Changes since delivery and by 67 patients for Movement during chewing and speaking. An affirmative "yes" answer was given by 1 patient for General problems and by 2 patients each for Changes since delivery and patients Movement during chewing and speaking.

Table 2 presents an overview of the responses to the categorical questions. Fisher's exact test yielded values of $\mathrm{p}>$ 0.05 within the patient groups and hence no significant influence of the location of the prosthesis on the occurrence of General problems, Changes since delivery, or Movement during chewing and speaking.

\section{Discussion}

The evaluation of the results showed that there was no statistically significant influence of the location of the prostheses (MX+MD, MX only, MD only) on any of the parameters. When considering the results, it should be noted that the patients were not interviewed after a defined period following delivery of the prostheses, resulting in different wearing times.

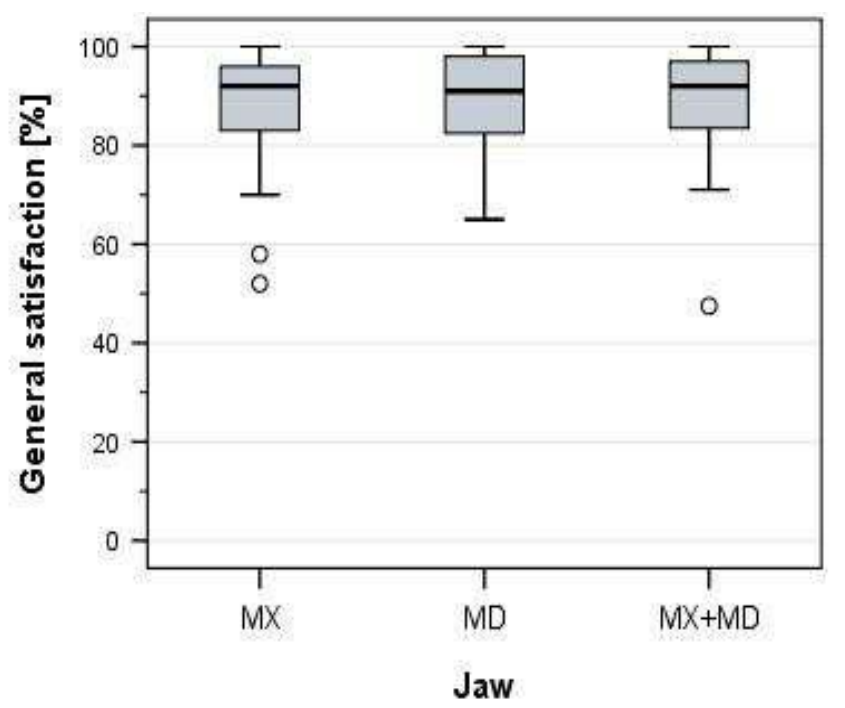

Figure 1: Assessment of general satisfaction.

Furthermore, the nature of the abutments was not linked to the evaluations. In addition, unlike other studies of patient satisfaction, the present one featured no control group, which should be viewed critically; nevertheless, the cases examined here can easily be aligned with those in other studies $[4,5,21]$. The method of surveying and evaluating patients using a visual analog scale (VAS) has been commonly used in other studies to assess patient satisfaction and to assess various parameters $9,10,12,16$.

The data showed prosthesis retention at levels of 87.96 $\pm 19.3 \%, 90.36 \pm 16.88 \%$, and $89.79 \pm 12.68 \%$ for the $\mathrm{MX}+\mathrm{MD}$, $\mathrm{MX}$ only, and MD only patient groups, respectively. Considering that the evaluated prostheses had ben in situ for between 18 months and 16 years at the time of the survey, this result underlines the good and consistent retention of the CEP. This favorable result was made possible by the functional principle of the CEP, which facilitates the creation of capillary forces within the narrow gap between the primary and secondary crowns. Due to the absence of frictional surfaces and frictional forces, in contrast to conventional double-crown systems, no retention losses occur; according to Cepa et al., such retention losses have a negative impact on patient satisfaction with doublecrowned prostheses [7]. The high and stable retention of the CEP according to Weigl has also been confirmed by Bayer et al., who showed a more stable and more reliable retention with zirconia primary crowns than with primary crowns made of precious alloys $[13,25]$. In addition, Weigl et al. found no signs of wear with a combination of a zirconia primary crownand an electroplated secondary crown, which underlines the durability of the materials and the associated high and stable retention [14].

The durability of the retention is also manifested in the results for the changes since delivery parameter. Here, only $2.7 \%$ of patients had noted any changes; $82.4 \%$ had not. This was accompanied by high scores for Wearing comfort and Speech function. Although there was no statistical significance, 


\begin{tabular}{|l|l|l|l|}
\hline Parameter & $\mathrm{MX}$ & $\mathrm{MD}$ & $\mathrm{MX}+\mathrm{MD}$ \\
\hline VAS scores in \% & $\mathrm{n}=22$ & $\mathrm{n}=39$ & $\mathrm{n}=13$ \\
\hline Overall satisfaction & $87.77 \pm 13.59$ & $89.79 \pm 9.81$ & $87.88 \pm 14.89$ \\
\hline Handling & $92.9 \pm 7.47$ & $90.85 \pm 8.62$ & $90.12 \pm 14.4$ \\
\hline Retention & $90.36 \pm 16.88$ & $89.79 \pm 12.68$ & $87.96 \pm 19.33$ \\
\hline Wearing comfort & $94.77 \pm 7.11$ & $90.95 \pm 9.48$ & $86.42 \pm 17.79$ \\
\hline Esthetics & $94 \pm 6.32$ & $88.36 \pm 13.09$ & $86.15 \pm 16.42$ \\
\hline Chewing function & $88.5 \pm 15.72$ & $90.23 \pm 8.93$ & $82.23 \pm 22.53$ \\
\hline Stability & $90.54 \pm 14.02$ & $87.44 \pm 13.22$ & $79.42 \pm 26.36$ \\
\hline Perception of security & $92.54 \pm 12.11$ & $91.026 \pm 7.34$ & $85.08 \pm 22.14$ \\
\hline Speech function & $90.23 \pm 12.72$ & $91.21 \pm 9.76$ & $84.38 \pm 20.66$ \\
\hline
\end{tabular}

Table 1. Overview of the VAS scores

\begin{tabular}{|l|l|l|l|l|l|l|}
\hline Parameter & no & $\begin{array}{l}\text { MX+MD - MX only } \\
- \text { MD only }\end{array}$ & neither & $\begin{array}{l}\text { MX+MD - MX } \\
\text { only - MD only }\end{array}$ & yes & $\begin{array}{l}\text { MX+MD - MX } \\
\text { only - MD only }\end{array}$ \\
\hline $\begin{array}{l}\text { General prob- } \\
\text { lems }\end{array}$ & 61 & $10 / 19 / 32$ & 12 & $3 / 3 / 6$ & 1 & $0 / 0 / 1$ \\
\hline $\begin{array}{l}\text { Changes since } \\
\text { delivery }\end{array}$ & 61 & $11 / 17 / 33$ & 11 & $1 / 5 / 5$ & 2 & $1 / 0 / 1$ \\
\hline $\begin{array}{l}\text { Movement dur- } \\
\text { ing chewing and } \\
\text { speaking }\end{array}$ & 67 & $12 / 19 / 36$ & 5 & $0 / 3 / 2$ & 2 & $1 / 0 / 1$ \\
\hline
\end{tabular}

Table 2: Overview of the responses to the categorical questions

these parameters show less favorable scores for patients with both maxillary and mandibular prostheses. This can also be due to the greater treatment effort and complexity and a greater need for follow-up care than in patients with prostheses in only one jaw.

Chewing function also received high scores. Here, too, less favorable scores were obtained for patients with both maxillary and mandibular prostheses. The reason for this could be that the need for a simultaneous rehabilitation of the maxilla and mandible is associated with a complete loss of denture support zones. In these cases, the practitioner must obtain a new jaw relation record, which can result in a new bite situation that also influences the chewing function.

In addition, the evaluations showed that the handling of the prostheses was rated as good, despite the strong denture retention. The underlying functional principle of the CEP provides for a steady and uniform insertion and removal force, which may explain the favorable Handling scores. This is supported by the results for General problems, which also includes the evaluation of problems involved in inserting and removing the prosthesis. Here, 61 of the 74 patients reported no problems.Only one patient examined general problems after incorporation of the denture. Here, the removal of the prostesis was describedas difficult. The remaining number of 12 patients classified this question as not evaluable.
Sense of security was evaluated at $85.08 \pm 22.14 \%, 92.54 \pm$ $12.11 \%$, and $91.03 \pm 7.34 \%$ for the MX+MD, MX only, and $\mathrm{MD}$ only patient groups, respectively. These results show that the patients feel safe with their prostheses, which is further supported by the results for Movement during chewing and speaking, where 2 patients gave an affirmative response. A negative response for Movement during chewing and speaking was proffered by $90.5 \%$, which highlights the good and secure fit of the prostheses.

The favorable results for the mentioned parameters were reflected by the uniformly high scores for Overall satisfaction, which were $87.88 \%, 87.77 \%$ and $89.79 \%$ for the $\mathrm{MX}+\mathrm{MD}, \mathrm{MX}$ only, and MD only patient groups, respectively. This is supported by a study by Grossmann et al., who also described a high level of overall satisfaction with double-crown prostheses [14].

The results obtained in the present study agree very well with the existing literature and show high levels of patient satisfaction with their CEP. This satisfaction is based on high retention, ease of handling, a high sense of security, and a low susceptibility to changes since delivery and general problems. There is no significant correlation between the results and the location of the prostheses. Thus, dentures retained by ceramic primary and electroplated secondary conical double crowns can be recommended for oral rehabilitationin the maxilla and mandible or both, from the dentist's as well as from the patient's point of view, if a clinical indication exists. 


\section{Acknowledgments} this study.

The authors report no conflicts of interest related to

\section{References}

1)

A. Al-Asfour, M. Waheedi, and S. Koshy (2018) Survey of Patient Experiences of Orthognathic Surgery: Health-Related Quality of Life and Satisfaction. Int J Oral Maxillofac Surg.

2) P. F. Allen, A. S. McMillan (2003) A Longitudinal Study of Quality of Life Outcomes in Older Adults Requesting Implant Prostheses and Complete Removable Dentures. Clin Oral Implants Res. 14:173-179.

3) M. A. Awad, J. P. Lund, E. Dufresne, et.al (2003) 'Comparing the Efficacy of Mandibular Implant-Retained Overdentures and Conventional Dentures among Middle-Aged Edentulous Patients: Satisfaction and Functional Assessment'. Int J Prosthodont. 16:117-122.

4) M. A. Awad, J. P. Lund, S. H. Shapiro et.al (2003). S. Feine, 'Oral Health Status and Treatment Satisfaction with Mandibular Implant Overdentures and Conventional Dentures: A Randomized Clinical Trial in a Senior Population'. Int J Prosthodont. 16:390-396.

5) J. Bouma, L. M. Boerrigter, R. P. Van Oort, et.al (1997) 'Psychosocial Effects of Implant-Retained Overdentures'. Int J Oral Maxillofac Implants. 12:515-22

6) M. Brennan, F. Houston, M. O'Sullivan, et.al (2010) 'Patient Satisfaction and Oral Health-Related Quality of Life Outcomes of Implant Overdentures and Fixed Complete Dentures'. Int J Oral Maxillofac Implants. 25:791-800.

7) S. Cepa, B. Koller, B. C. Spies, et.al (2017) 'Implant-Retained Prostheses: Ball Vs. Conus Attachments a Randomized Controlled Clinical Trial'. Clin Oral Implants Res. 28:177-185.

8) P. Ceruti, S. R. Bryant, J. H. Lee, et.al (2010) 'Magnet-Retained Implant-Supported Overdentures: Review and 1-Year Clinical Report'. J Can Dent Assoc. 76: a52.

I. J. De Kok, K. H. Chang, T. S. Lu, and L. F. Cooper (2011) 'Comparison of Three-Implant-Supported Fixed Dentures and Two-Implant-Retained Overdentures in the Edentulous Mandible: A Pilot Study of Treatment Efficacy and Patient Satisfaction', Int J Oral Maxillofac Implants. 26: 415-246.

10)

A. M. El-Sheikh, O. F. Shihabuddin, and S. M. Ghoraba (2012) 'A Prospective Study of Early Loaded Single Implant-Retained Mandibular Overdentures: Preliminary One-Year Results'. Int J Dent. 23:64-09.
11)

J. S. Ellis, G. Burawi, A. Walls, and J. M. Thomason (2009) 'Patient Satisfaction with Two Designs of Implant Supported Removable Overdentures; Ball Attachment and Magnets'. Clin Oral Implants Res. 20:1293-128.

J. S. Ellis, N. D. Pelekis, and J. M. Thomason (2007) 'Conventional Rehabilitation of Edentulous Patients: The Impact on Oral Health-Related Quality of Life and Patient Satisfaction'. J Prosthodont. 16:37-42. A. E. Gerritsen, P. F. Allen, D. J. Witter, et.al (2010) 'Tooth Loss and Oral Health-Related Quality of Life: A Systematic Review and Meta-Analysis', Health Qual Life Outcomes. 8:126.

14) A. C. Grossmann, A. J. Hassel, O. Schilling, F. Lehmann, et.al (2007) 'Treatment with Double Crown-Retained Removable Partial Dentures and Oral Health-Related Quality of Life in Middle- and High-Aged Patients', Int J Prosthodont. 20:576-578.

15)

J. Montero, R. Castillo-Oyague, C. D. Lynch, et.al (2013) 'Self-Perceived Changes in Oral Health-Related Quality of Life after Receiving Different Types of Conventional Prosthetic Treatments: A Cohort Follow-up Study', J Dent. 41:493-503.

16)

M. C. Nunez, D. C. Silva, B. A. Barcelos, et.al (2015) 'Patient Satisfaction and Oral Health-Related Quality of Life after Treatment with Traditional and Simplified Protocols for Complete Denture Construction', Gerodontology. 32:247253.

17)

A. Preciado, J. Del Rio, M. J. Suarez-Garcia, et.al (2012) 'Differences in Impact of Patient and Prosthetic Characteristics on Oral Health-Related Quality of Life among Implant-Retained Overdenture Wearers'. J Dent. 40:857-865.

18) M. Sanchez-Siles, J. F. Ballester-Ferrandis, N. Salazar-Sanchez, et.al (2017) 'Long-Term Evaluation of Quality of Life and Satisfaction between Implant Bar Overdentures and Conventional Complete Dentures: A 23 Years Retrospective Study'. Clin Implant Dent Relat Res.

19)

H. Tan, K. G. Peres, and M. A. Peres (2016) 'Retention of Teeth and Oral Health-Related Quality of Life', J Dent Res. 95:1350-1357.

20)

J. M. Thomason (2010) 'The Use of Mandibular Implant-Retained Overdentures Improve Patient Satisfaction and Quality of Life'. J Evid Based Dent Pract. 10:61-63.

J. M. Thomason, J. P. Lund, A. Chehade, and J. S. Feine (2003) 'Patient Satisfaction with Mandibular Implant Overdentures and Conventional Dentures 6 Months after Delivery'. Int J Prosthodont. 16: 467-473. 
22)

S. Wallace, S. Samietz, M. Abbas, et.al (2018)

'Impact of Prosthodontic Rehabilitation on the Masticatory Performance of Partially Dentate Older Patients: Can It Predict Nutritional State? Results from a Rct'. J Dent. 68: 66-71. P. Weigl, and H. C. Lauer (2000) 'Advanced Biomaterials Used for a New Telescopic Retainer for Removable Dentures', J Biomed Mater Res. 53:337-347.
Submit your manuscript to a JScholar journal and benefit from:

๑ Convenient online submission

- Rigorous peer review

- Immediate publication on acceptance

ब Open access: articles freely available online

I High visibility within the field

ब Better discount for your subsequent articles Submit your manuscript at http://www.jscholaronline.org/submit-manuscript.php 